\title{
Dark matter density profiles: a comparison of nonextensive theory with $\mathbf{N}$-body simulations
}

\author{
T. Kronberger ${ }^{1,2}$, M. P. Leubner ${ }^{1}$, and E. van Kampen ${ }^{1}$ \\ ${ }^{1}$ Institut für Astro- und Teilchenphysik, Leopold-Franzens-Universität Innsbruck, Technikerstr. 25, 6020 Innsbruck, Austria \\ e-mail: Thomas.Kronberger@uibk . ac . at \\ 2 Institut für Astrophysik, Universität Göttingen, Friedrich-Hund-Platz 1, 37077 Göttingen, Germany \\ Received 30 November 2005 / Accepted 11 March 2006
}

\section{ABSTRACT}

\begin{abstract}
Density profiles of simulated galaxy cluster-sized dark matter haloes are analysed in the context of a recently introduced nonextensive theory of dark matter and gas density distributions. Nonextensive statistics accounts for long-range interactions in gravitationally coupled systems and is derived from the fundamental concept of entropy generalization. The simulated profiles are determined down to radii of $\approx 1 \%$ of $\mathrm{R}_{200}$. The general trend of the relaxed, spherically averaged profiles is accurately reproduced by the theory. For the main free parameter $\kappa$, measuring the degree of coupling within the system, and linked to physical quantities as the heat capacity and the polytropic index of the self-gravitating ensembles, we find a value of -15 . The significant advantage over empirical fitting functions is provided by the physical content of the nonextensive approach.
\end{abstract}

Key words. cosmology: theory - cosmology: dark matter - methods: $N$-body simulations - galaxies: clusters: general

\section{Introduction}

The radial density profiles of dark matter (DM) haloes, generated in the process of hierarchical structure formation, were modelled in the past primarily on phenomenological grounds. Highresolution $N$-body simulations were performed (e.g. Navarro et al. 1996, 1997; Moore et al. 1999) in order to reveal the DM density distribution. The functional dependence $\rho_{\mathrm{DM}} \propto$ $\left(r / r_{\mathrm{S}}\right)^{-\alpha}\left(1+r / r_{\mathrm{S}}\right)^{-(3-\alpha)}$ (Zhao 1996) provides reasonable fits to haloes on all observable scales (e.g. Ricotti 2003). Here $r_{\mathrm{S}}$ is a scaling radius and the parameter $\alpha$ is related to the initial power spectrum. For $\alpha=1$ this expression represents the Navarro et al. $(1996,1997)$, hereafter NFW profile, while the Moore et al. (1999) profile follows with $\alpha=1.5$. Both on theoretical and on observational grounds there is still profound discussion on the innermost slope of the DM density profile. While numerical simulations generally predict a divergent, "cuspy" behaviour towards the centre (e.g. Navarro et al. 2004) observations favour a flat, cored profile (e.g. Gentile et al. 2004 and references therein).

Only a few attempts to provide theoretically motivated models for density profiles of cosmic structure were proposed so far. Early analytic work was pioneered by Gunn \& Gott (1972) and subsequently elaborated by Fillmore \& Goldreich (1984) and Bertschinger (1985), who derived similarity solutions for the self-similar collapse of spherical perturbations. Those solutions were naturally power-laws for virialised objects. Later, correlations between the slope of the density profile and the form of the power spectrum of the initial density perturbations were recognized (e.g. Hoffman 1988). More recent attempts based on analytic infall models were provided by Williams et al. (2004) and Ascasibar et al. (2004) while Hansen (2004) and Austin et al. (2005) used the Jeans equation to gain further insight.

In this work we apply a recently proposed nonextensive theory for DM and gas density profiles (Leubner 2005), accounting for long-range interactions and correlations present in any astrophysical system, to results obtained from $\mathrm{N}$-body simulations. The term "nonextensive" is used for statistical ensembles, whose total entropy is not an additive quantity as in standard Boltzmann-Gibbs-Shannon (BGS) thermo-statistics. Instead, pseudo-additive entropy generalization accounts for correlations between sub-systems. The possibility to describe DM density profiles using nonextensive statistics was recently also suggested (Hansen et al. 2005) in the context of Eddingtons formula (e.g. Binney \& Tremaine 1994), whereas an earlier theoretical link between entropy generalization and self-gravitating structures was provided by Plastino \& Plastino (1993).

The classical BGS extensive thermo-statistics constitutes a powerful tool when microscopic interactions and memory are short-ranged and the environment is an Euclidean space-time, a continuous and differentiable manifold. However, in the present situation we are dealing with astrophysical systems, generally subject to spatial or temporal long-range interactions, i.e. ensembles evolving under correlations that make their behavior nonextensive. A suitable generalization of the BGS entropy for statistical equilibrium was first proposed by Renyi (1955) and subsequently by Tsallis (1988), preserving the usual properties of positivity, equiprobability and irreversibility, but suitably extending the standard extensivity or additivity to nonextensivity. The main theorems of the classical Maxwell-Boltzmann statistics admit profound generalizations within nonextensive statistics (sometimes referred to as q-statistics where $q$ characterizes the degree of nonextensivity of the system), wherefore a variety of subsequent analyses were devoted to clarify the mathematical and physical consequences of pseudo-additivity, for an early review see e.g. Tsallis (1995). Those include a reformulation of the classical $N$-body problem within the extended statistical mechanics (Plastino et al. 1994) and the development of nonextensive distributions (Silva et al. 1998; Almeida 2001). A deterministic connection between the generalized entropy and the resulting power-law functionals was recognized 
(Andrade et al. 2002), as well as the dual structure of nonextensive statistical theory (Karlin et al. 2002).

Besides new insights into fundamental physics astrophysical applications support q-non-extensive statistics, proposing distribution functions for stellar polytropes (Plastino et al. 1993) and explaining the solar neutrino counting rate (Kaniadakis et al. 1996). Cosmological tests provide a bound on the degree of possible non-extensivity from primordial helium abundance data (Torres et al. 1997) and the temperature of the cosmic microwave background radiation in a Robertson-Walker universe was shown to be independent of the degree of non-extensivity (Hamity et al. 1996). Furthermore, generalized statistics was applied to large scale astrophysical systems subject to longrange gravitational forces in view of galaxy distribution studies (Nakamichi et al. 2002) and reformulated also in the context of special relativity (Kaniadakis 2002). Recently nonextensive theory was successfully introduced also to study the scale dependence of intermittent flows in astrophysical plasma turbulence, appearing as consequence of long-range interactions (Leubner \& Vörös 2005). In this context the resulting bi-kappa distribution function appears as manifestation of the dual nature of nonextensive statistics, which provides also the physical background of entropy bifurcation in the theoretical context of DM and gas density distributions of clustered matter (Leubner 2005).

We relate in the following nonlocality in gravitationally clustered astrophysical structures to the presence of long-range forces in nonextensive systems and demonstrate that density distributions derived within the framework of entropy generalization consistently reproduce the density profile of cluster-sized dark matter haloes forming in $\Lambda$ cold dark matter $(\Lambda \mathrm{CDM})$ $N$-body simulations. This result is furthermore supported by the integrated mass profile of an observed relaxed galaxy cluster. Consequently, nonextensive statistics provides a physically interpretable alternative to empirical fitting procedures.

\section{Theory}

Let us first illuminate the property of pseudo-additivity in the context of nonextensive entropy generalization by considering two sub-systems $A$ and $B$ such that

$S_{\kappa}(A+B)=S_{\kappa}(A)+S_{\kappa}(B)+\frac{1}{\kappa} S_{\kappa}(A) S_{\kappa}(B)$

where $S_{K}$ denotes the entropy as depending on the entropic index $\kappa$, a parameter specifying the degree of nonextensivity in the system. For $\kappa=\infty$ the last term on the right hand side cancels leaving the additive entropy of the standard BGS statistics. Hence, nonlocality or long-range interactions are introduced by the multiplicative last term on the right hand side of Eq. (1) accounting for correlations between the subsystems $A$ and $B$. As measure of the entropy mixing the parameter $\kappa$ quantifies the degree of statistical correlations in the system and thus accounts for nonlocality and long-range interactions or couplings, respectively. In general, the pseudo-additive, $\kappa$-weighted term may assume positive or negative definite values indicating a nonextensive entropy bifurcation. Obviously, nonextensive systems are subject to a dual nature since positive $\kappa$-values imply the tendency to less organized states where the entropy increases whereas negative $\kappa$-values provide a higher organized state of decreased entropy, see Leubner (2005).

Next, consider a DM halo as a self-gravitating collisionless system of particles in dynamical equilibrium (e.g. Burkert 2000; Firmani et al. 2000). Consistent with Eq. (1) the corresponding generalized entropy $S(\kappa)$, characterising systems subject to longrange interactions and couplings in nonextensive statistics, reads (Tsallis 1988; Leubner 2004)

$S_{\kappa}=\kappa k_{\mathrm{B}}\left(\sum p_{i}^{1-1 / \kappa}-1\right)$

where $p_{i}$ is the probability of the $i$ th microstate, $k_{\mathrm{B}}$ is Boltzmann's constant. The transformation $\kappa=1 /(1-q)$ links the $\kappa$-formalism, commonly applied in astrophysical plasma modelling to the Tsallis q-statistics (Leubner 2002). Here $\kappa=\infty$ represents the extensive limit of statistical independence and recovers the classical BGS entropy as $S_{B}=-k_{B} \sum p_{i} \ln p_{i}$.

Since entropy and probability distributions reside physically on the same level the corresponding generalized energy distribution, accounting for long-range interactions, is available. In Maxwells derivation the velocity components of the distribution $f(\boldsymbol{v})$ are uncorrelated where $\ln f$ can be expressed as a sum of the logarithms of the one dimensional distribution functions. In nonextensive systems one needs to keep correlations between the components, which can be done conveniently by extremizing the entropy under conservation of mass and energy yielding the corresponding one- and three-dimensional power-law distributions in velocity space (Leubner 2004)

Here we must retain the spatial dependence and consider a spherical symmetric, self-gravitating and collisionless $N$-body system where the corresponding steady state phase-space distribution $f(r, v)$ obeys the Vlasov equation. If the system of particles itself provides the gravitational potential $\Phi$ and $f(r, v)$ is regarded as the mass distribution then Poisson's equation reads

$\Delta \Phi=4 \pi G \rho=4 \pi G \int f\left(\frac{1}{2} v^{2}+\Phi\right) \mathrm{d}^{3} v$.

This representation governs the equilibrium of the system where commonly the relative particle energy $E_{r}=-1 / 2 v^{2}+\Psi$ is introduced (e.g. Binney \& Tremaine 1994).

Extremizing the generalized entropy functional (2) with regard to conservation of mass and energy (Plastino 1993) the resulting energy distribution $f^{ \pm}\left(E_{r}\right)$ involved in Eq. (3) reads

$f^{ \pm}\left(E_{r}\right)=B^{ \pm}\left[1+\frac{1}{\kappa} \frac{v^{2} / 2-\Psi}{\sigma^{2}}\right]^{-\kappa}$.

The superscripts refer to the positive or negative intervals of the entropic index $\kappa$, accounting for less (+) and higher (-) organized states and thus reflecting the accompanying entropy increase or decrease, respectively, (see Leubner 2005). $\sigma$ represents the mean energy or variance of the distribution and $B^{ \pm}$denote the corresponding normalisation constants depending on the entropic index $\kappa$, for details see Leubner (2004). For $\kappa \rightarrow \infty$ Eq. (4) approaches the exponential distribution function defining the density profile of the isothermal sphere (Binney \& Tremaine 1994).

After incorporating the sign of $\kappa$ into Eq. (4), we perform separately for positive and negative definite $\kappa$ the integration over all velocities where $B^{ \pm}$must be used consistently. The resulting solution provides the relation for the density evolution of a system subject to long range interactions in a gravitational potential as

$\rho^{ \pm}=\rho_{0}\left[1-\frac{1}{\kappa} \frac{\Psi}{\sigma^{2}}\right]^{3 / 2-\kappa}$.

Equation (5) generates for finite positive values of $\kappa$ pronounced density tails, whereas for negative $\kappa$-values the solutions are restricted within the cutoff at $\kappa=\Psi / \sigma^{2}$ and $\kappa=-\infty$. 


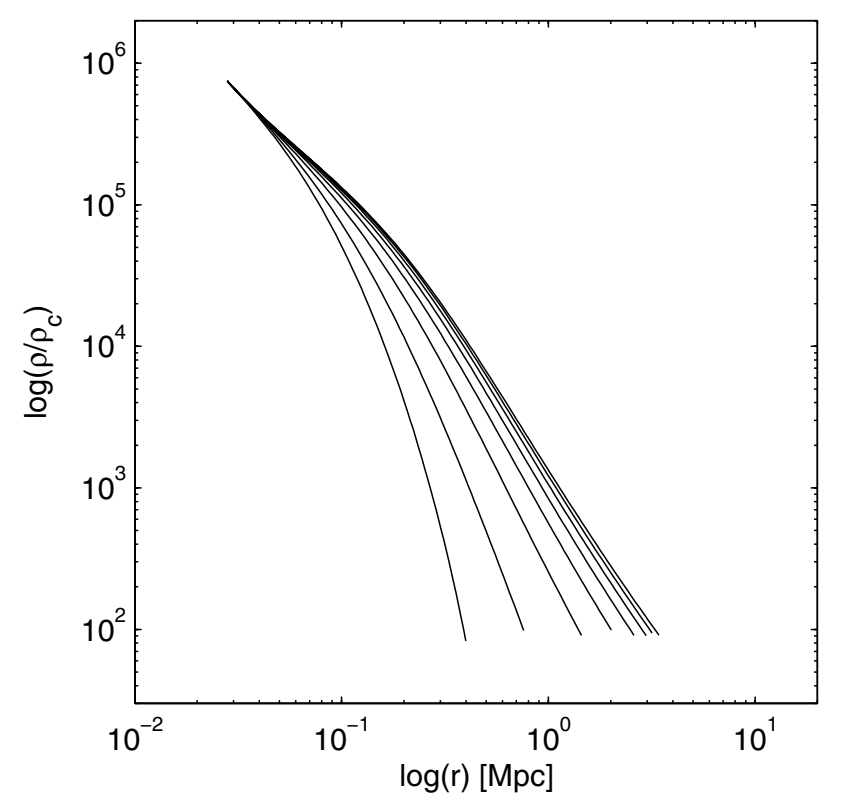

Fig. 1. Characteristics of the radial DM density profile: A sequence of solutions to Eq. (6) is plotted corresponding to different values of the parameter $\kappa$, varied from -2 to $-\infty$ (left to right). The second free parameter $\sigma$ was kept fixed for this schematic plot.

The duality of equilibria in nonextensive statistics is manifest in two families, the nonextensive thermodynamic equilibria and the equilibria of kinetic equations, where positive $\kappa$-values correspond to the stationary states of thermodynamics and negative $\kappa$-values to kinetic stationary states (Karlin et al. 2002). The limiting BGS state for $\kappa=\infty$ is characterized by self-duality. Physically the parameter $\kappa$ is related to the heat capacity of the medium where negative heat capacity, corresponding to values $\kappa<0$, is a typical property of self-gravitating systems, see e.g. Firmani et al. (2000). The nonextensive bifurcation into two distributions $f^{ \pm}\left(E_{r}\right)$ or $\rho^{ \pm}$, respectively, requires to identify the density profile (5) for positive definite $\kappa$ as the proper distribution of the thermodynamic state of the gas, whereas the negative definite counterpart is associated with the self-gravitating DM distribution, we will focus on. In the limit $\kappa \rightarrow \infty$ both solutions merge at the isothermal sphere solution, defined by the limiting exponential distribution obtained for $\kappa=\infty$ in Eq. (4).

Finally, we combine Poisson's equation $\Delta \Psi=-4 \pi G \rho$ and the density distribution Eq. (5), which yields after re-arranging for $\Psi$ a second order nonlinear differential equation for the variation of a spherically symmetric gas and DM distribution as (Leubner 2005)

$\frac{\mathrm{d}^{2} \rho}{\mathrm{d} r^{2}}+\frac{2}{r} \frac{\mathrm{d} \rho}{\mathrm{d} r}-\left(1-\frac{1}{n}\right) \frac{1}{\rho}\left(\frac{\mathrm{d} \rho}{\mathrm{d} r}\right)^{2}-\frac{4 \pi G n}{(3 / 2-n) \sigma^{2}} \rho^{2}\left(\frac{\rho}{\rho_{0}}\right)^{-1 / n}=0(6)$

where $n=3 / 2-\kappa$ is introduced and corresponds to the polytropic index of stellar dynamical systems. For negative definite $\kappa$-values the DM density profiles defined by Eq. (6) are found numerically by solving the corresponding two first order differential equations with a Runge-Kutta routine. Figure 1 shows the characteristics of the radial density profile as depending on the variation of the entropic index $\kappa$ and computed from Eq. (6) for DM profiles. With increasing $\kappa$ the solution converges from left to right to the isothermal sphere density profile, an environment subject to statistical independence of the constituents.

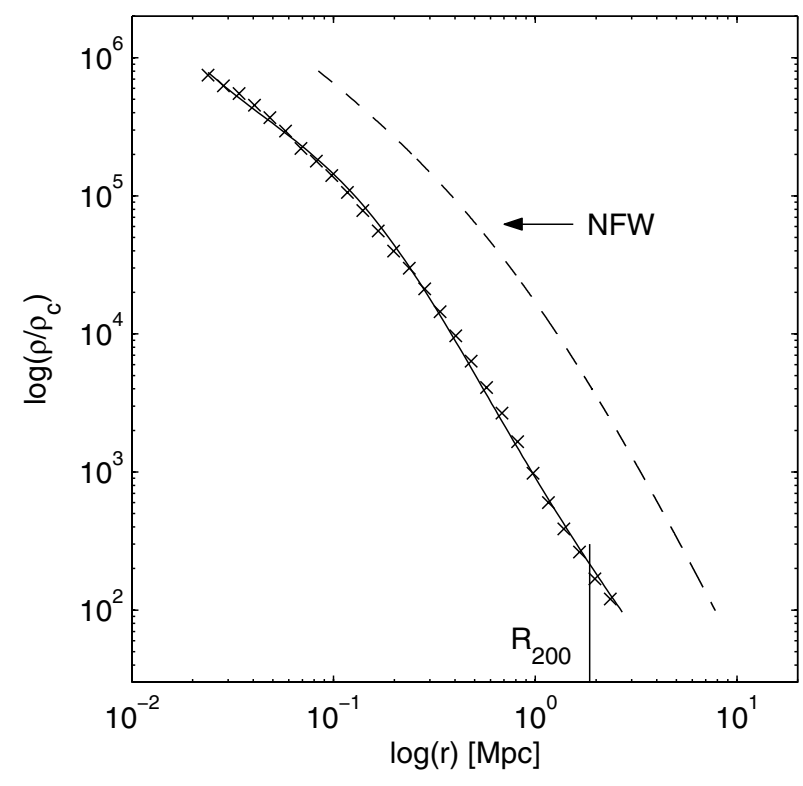

Fig. 2. Radial dark matter density profile obtained from the simulation (crosses). The solid line shows a fit of the nonextensive theory to the data with best fitting values of $\kappa=-15$ and $\sigma=0.12$. For comparison, also the best fitting NFW profile is provided as a dashed line but shifted to the right for better visibility. $R_{200}$ indicates the virial radius.

\section{3. $\mathbf{N}$-body simulations}

The numerical simulations were performed using an adapted version of the treecode of Barnes \& Hut (1986), using $128^{3}$ particles in a comoving volume of $\left(32 h^{-1} \mathrm{Mpc}\right)^{3}$ in a $\Lambda$ CDM cosmology with $\Omega_{\Lambda}=0.7, \Omega_{\mathrm{m}}=0.3, h=0.7, \sigma_{8}=0.93$. We employ the constrained random field technique of Hoffman \& Ribak (1991), as implemented by van de Weygaert \& Bertschinger (1996), to form a rich cluster in the centre of the simulation box. The (Plummer) softening length was set to $7 h^{-1} \mathrm{kpc}$, which also sets the spatial resolution of the simulation. Well over a thousand time steps were used to integrate the constrained initial conditions to the present epoch. The diagonal components of the velocity dispersion tensor for the mass within $3 \mathrm{Mpc}$ are: $\sigma_{x}=804 \mathrm{~km} \mathrm{~s}^{-1}, \sigma_{y}=872 \mathrm{~km} \mathrm{~s}^{-1}$, and $\sigma_{z}=1043 \mathrm{~km} \mathrm{~s}^{-1}$.

To obtain a spherically averaged mass density profile at each time step, we first determine the cluster centre using an iterative technique similar to the one presented in Power et al. (2003). We compute the centre of mass from particles within spheres of decreasing radius. Then we bin the particles logarithmically according to their distance from the cluster centre and calculate the density profile out to a radius of $\sim 2.5 \mathrm{Mpc}$ (physical distance). In order to get reliable results, we consider only scales larger than the softening length of the particles and the number of particles within each bin is always well above 100 .

\section{Discussion and conclusions}

With this setup we studied a sample of 3 cluster-sized DM haloes with different formation histories. We confirm the commonly known property that the inner density profile of the halo does not evolve anymore after a redshift of about one. We then use the relaxed density profile of the most massive cluster halo of our sample $\left(M_{\text {vir }}=8.2 \times 10^{14} M_{\odot}\right.$ within $R_{200}$ at $\left.z=0\right)$, which is also the most virialised system already at $z \approx 1$, and fit the nonextensive distribution by solving the differential Eq. (6). The result is shown in Fig. 2 together with the corresponding NFW profile for 


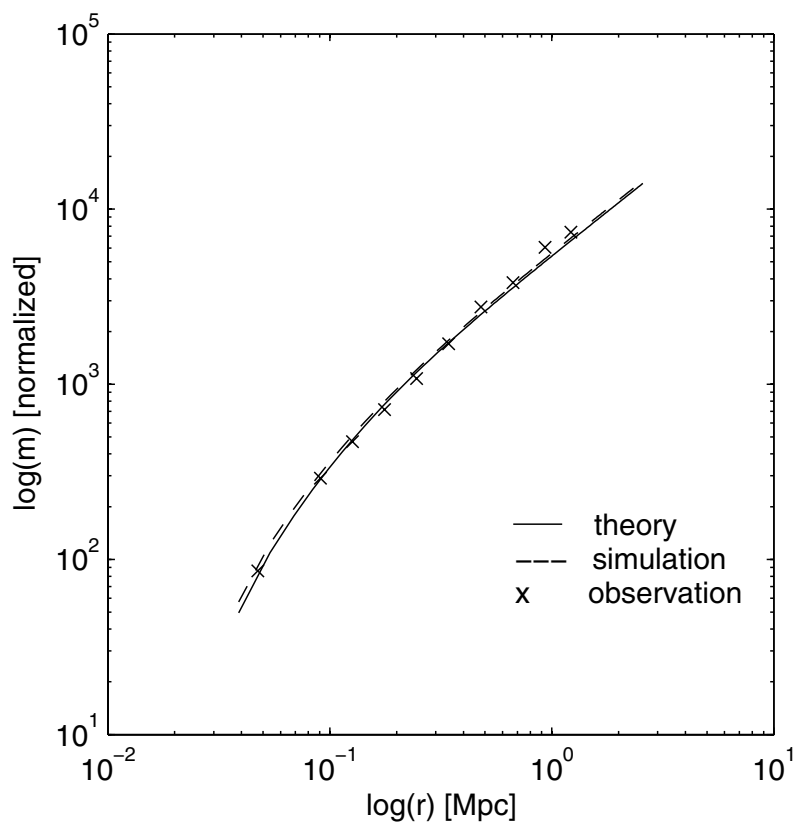

Fig. 3. Integrated mass profile of the galaxy cluster A1413 (crosses, Pointecouteau et al. 2005) compared to the simulated (dashed) and theoretical (solid) curve.

comparison. The nonextensive density profile follows the result of the simulation accurately at all cluster radii with best fitting values of $\kappa=-15$, indicating the presence of strong correlations in the system, and $\sigma=0.12$. Hansen et al. (2005) investigated possible theoretical constraints on the entropic index $q$, or equivalently $\kappa$. Arguing that negative velocity dispersions are difficult to interpret in a cosmological context, he arrived at the condition $q<5 / 3$, which reads in the $\kappa$ formalism $\kappa<-3 / 2$. By demanding a positive proportion between temperature and internal energy Boghosian (1999) arrived at the same constraint. Our best fitting value of $\kappa=-15$ clearly obeys this restriction.

Figure 2 demonstrates that also the empirical NFW profile represents the simulated radial density distribution well. However, the use of the nonextensive approach allows one to interpret differences that might occur, e.g. in comparison to observations, within the physical context of entropy generalization. On the other hand, in deriving Eq. (6) we assumed that the phasespace distribution function $f(r, v)$ is a function of energy only. This is essentially true for systems having an isotropic velocity dispersion tensor. If this is not the case, as suggested by numerous numerical simulations (e.g. Cole \& Lacey 1996; Natarajan et al. 1997), $f(r, v)$ is generally also a function of the angular momentum vector $\boldsymbol{L}$ of the system. Therefore, for systems with a highly anisotropic velocity dispersion, solutions of Eq. (6) might not represent the density profile in detail. Furthermore, there is no physical reason to keep $\kappa$ constant, as used within the present analysis. For instance, one might suspect $\kappa$ to vary with radius, as the strength of the correlations could be a function of the distance. However, since the shape of the density profiles, from simulations and observations, are reproduced accurately within the applied simplifications (see Figs. 2 and 3), and given the fact that also the cluster halo shown in Fig. 2 is not completely isotropic (see Sect. 4), modifications due to these open issues are expected to be small. In addition, presently the resolution of the simulation is insufficient to obtain robust estimates for the density distribution at smaller radii than presented in Fig. 2. However, from high resolution simulations (e.g. Navarro et al. 2004) it is known that DM profiles do not become flat at currently probed radii $\left(<1 \%\right.$ of $\left.R_{200}\right)$. Finally, in Fig. 3 the present analysis is tested on an integrated mass profile evaluated from X-ray observations. Along with the integrated simulation profile and the nonextensive solution a normalized profile of the galaxy cluster A1413 is plotted (Pratt \& Arnaud 2002; Pointecouteau et al. 2005). In the observable radial range both, the simulated and the nonextensive profile, perfectly match the observed data set. Note that A1413 is a relaxed cluster with no cooling core, consistent with the theoretical context restricted to equilibrium states.

In principle, as the topology of the solutions to Eq. (6) is very complex, also density profiles from systems in earlier evolutionary stages can be well reproduced. However, in deriving the differential Eq. (6) we implied that the system is relaxed, as we extremized the generalized entropy functional (2) to get the equilibrium phase space distribution function. As the cluster is not relaxed at redshifts $z>1$ the physical interpretation of the fitting parameters fails or is extremely complicated in this redshift range.

We also note that the strength of correlations within a system depends on the cosmological model, or more precisely on the form of the power spectrum of the initial density fluctuations, i.e. $P(k) \propto k^{n}$. It has been recognized already in early numerical works that increasing $n$ leads to a steepening of the density profiles (e.g. Hoffman 1988; Efstathiou et al. 1988). Our theoretical framework of non-extensive statistics and the derivation of Eq. (6) do not depend on the initial power spectrum. Therefore the theory is also valid for different power spectra than the one adopted for this work $(n=1)$. Even without performing additional simulations ourselves, we can conclude that long-range interactions are more important for high spectral indices $n$, as steeper profiles are realised by lower values of $\kappa$.

In conclusion, nonextensive statistics provides on physical grounds access to the study of DM density profiles in relaxed clusters and is able to model the corresponding equilibrium states of self gravitating collisionless systems. The significant advantage over empirical fitting functions is provided through the physical content of the parameters involved in the nonextensive approach, with $\kappa$ as a measure of the degree of correlations in the system and $\sigma$, the characteristic energy. Links between the entropic index $\kappa$, the heat capacity and the polytropic index of DM haloes are subject of a further study.

Acknowledgements. The authors acknowledge the German Science Foundation (DFG), through grant Zi 663/6-1, the Austrian Science Foundation (FWF), through grant P15868 and the UniInfrastrukturprogramm 2004 des bm:bwk Forschungsprojekt Konsortium Hochleistungsrechnen. We are grateful to the anonymous referee for his/her criticism that helped to improve the paper. Joshua Barnes and Piet Hut are gratefully acknowledged for use of their treecode, and Edmund Bertschinger and Rien van de Weygaert for use of their constrained initial conditions code. Etienne Pointecouteau, Gabriel Pratt and Monique Arnaud are gratefully acknowledged for providing the data of the integrated mass profile of the cluster A1413.

\section{References}

Almeida, M. P. 2001, Physica A, 300, 424

Andrade, J. S., Almeida, M. P., Moreira, A. A., \& Farias, G. A. 2002, Phys. Rev. E, 65, 036121

Ascasibar, Y., Yepes, G., Gottlöber, S., \& Müller, V. 2004, MNRAS, 352, 1109 Austin, C. G., Williams, L. L. R., Barnes, E. I., Babul, A., \& Dalcanton, J. J. 2005, ApJ, 634, 756

Barnes, J., \& Hut, P. 1986, Nature, 324, 446

Bertschinger, E. 1985, ApJS, 58, 39

Binney, J., \& Tremaine, S. 1994, Galactic Dynamics (Princeton Univ. Press) 
Boghosian, B. M. 1999, Brazilian J. Phys., 29, 91

Burkert, A. 2000, ApJ, 534, L143

Cole, S., \& Lacey, C. 1996, MNRAS, 281, 716

Efstathiou, G., Frenk, C. S., White, S. D. M., \& Davis, M. 1988, MNRAS, 235, 715

Fillmore, J. A., \& Goldreich, P. 1984, ApJ, 281, 1

Firmani, C., Onghia, E. D., Avila-Reese, V., Chincarini, G., \& Hernandez, X. 2000, MNRAS, 315, L29

Gentile, G., Salucci, P., Klein, U., Vergani, D., \& Kalberla, P. 2004, MNRAS, 351,903

Gunn, J. E., \& Gott, J. R. I. 1972, ApJ, 176, 1

Hamity, V. H., \& Barraco D. E. 1996, Phys. Rev. Lett., 76, 4664

Hansen, S. H. 2004, MNRAS, 352, L41

Hansen, S. H., Egli, D., Hollenstein, L., \& Salzmann, C. 2005, New Astron., 10, 379

Hoffman, Y. 1988, ApJ, 328, 489

Hoffman, Y., \& Ribak, E. 1991, ApJ, 380, L5

Kaniadakis, G. 2002, Phys. Rev. E, 66, 056125

Kaniadakis, G., Lavagno, A., \& Quarati, P. 1996, Phys. Lett. B, 369, 308

Karlin, I. V., Grmela, M., \& Gorban, A. N. 2002, Phys. Rev. E, 65, 036128

Leubner, M. P. 2002, Ap\&SS, 282, 573

Leubner, M. P. 2004, ApJ, 404, 469

Leubner, M. P. 2005, ApJ, 632, L1
Leubner, M. P., \& Voeroes, Z. 2005, ApJ, 618, 547

Moore, B., Quinn, T., Governato, F., Stadel, J., \& Lake, G. 1999, MNRAS, 310, 1147

Nakamichi, A., Joichi, I., Iguchi, O., \& Morikawa, M. 2002, Chaos, Solitons \& Fractals, 13595

Natarajan, P., Hjorth, J., \& van Kampen, E. 1997, MNRAS, 286, 329

Navarro, J. F., Frenk, C. S., \& White, S. D. M. 1996, ApJ, 462, 563

Navarro, J. F., Frenk, C. S., \& White, S. D. M. 1997, ApJ, 490, 493

Navarro, J. F., Hayashi, E., Power, C., et al. 2004, MNRAS, 349, 1039

Plastino, A. R., \& Plastino, A. 1993, Phys. Lett. A, 174, 384

Plastino, A. R., Plastino, A., \& Tsallis, C. 1994, J. Phys. A: Math. Gen., 27, 5707

Power, C., Navarro, J. F., Jenkins, A., et al. 2003, MNRAS, 338, 14

Pointecouteau, E., Arnaud, M., \& Pratt, G. W. 2005, A\&A, 435, 1

Pratt, G. W., \& Arnaud, M. 2002, A\&A, 394, 375

Renyi, A. 1955, Acta Math. Hungaria, 6, 285

Ricotti, M. 2003, MNRAS, 344, 1237

Silva, R., Plastino, A. R., \& Lima, J. A. S. 1998, Phys. Lett. A, 249, 401

Torres, D. F., Vucetich, H., \& Plastino, A. 1997, Phys. Rev. Lett., 79, 1588

Tsallis, C. 1988, J. Stat. Phys., 52, 479

Tsallis, C. 1995, Physica A, 221, 277

van de Weygaert, R., \& Bertschinger, E. 1996, MNRAS, 281, 84

Williams, L. L. R., Babul, A., \& Dalcanton, J. J. 2004, ApJ, 604, 18

Zhao, H. 1996, MNRAS, 278, 488 